\title{
A SUBDIRECT DECOMPOSITION OF SEMIPRIME RINGS AND ITS APPLICATION TO MAXIMAL QUOTIENT RINGS
}

\section{LOUIS HALLE ROWEN ${ }^{1}$}

ABSTRACT. Levy [2] has examined semiprime rings which are irredundant subdirect products of prime rings. In this note we look at the role of inessential prime ideals and see how every semiprime ring is a subdirect product of (i) a semiprime ring which is an irredundant subdirect product of prime rings, and (ii) a semiprime (nonprime) ring, all of whose prime ideals are essential. This leads to a direct sum decomposition of maximal left quotient rings of semiprime rings with left singular ideal zero.

Let $R$ be a semiprime ring, i.e., $R$ has no nonzero nilpotent ideals. Let $\mathcal{P}=\{$ prime ideals of $R\}$. It is well known that $\bigcap\{P \in \mathscr{P}\}=0$. If $A$ is a subset of $R$ then let

$$
\text { Ann } A=\{r \in R \mid a r=0 \text { for all } a \in A\}
$$

and let

$$
\operatorname{Ann}^{\prime} A=\{r \in R \mid r a=0 \text { for all } a \in A\} .
$$

Since $R$ is semiprime, Ann $A=\operatorname{Ann}^{\prime} A$ for any ideal $A$ of $R$. (Proof. $\left(A \operatorname{Ann}^{\prime} A\right)^{2} \subseteq\left(A n n^{\prime} A\right) A=0$, so $A \operatorname{Ann}^{\prime} A=0$, implying $A n n^{\prime} A \subseteq$ Ann $A$; Ann $A \subseteq$ Ann' $A$ follows by symmetry.) We say an ideal $A$ is essential if $A \cap B \neq 0$ for any nonzero ideal $B$ of $R$. (Note. 0 is an inessential ideal.) This is equivalent to Ann $A=0$ by an argument similar to the previous one. We say an ideal $B$ of $R$ is semiprime if $R / B$ is a semiprime ring.

Lemma 1. If $B$ is an ideal of $R$ then Ann $B$ is a semiprime ideal of R. In fact, Ann $B=\bigcap\{P \in \mathscr{P} \mid B \underline{\perp} P\}$.

Proof. $B \subseteq \bigcap\{P \in \mathcal{P} \mid B \subseteq P\}$. Now let $B^{\prime}=\bigcap\{P \in \mathcal{P} \mid B \nsubseteq \subset P\} . B B^{\prime} \subseteq$ $B \cap B^{\prime}=0$, so $B^{\prime} \subseteq$ Ann $B$. On the other hand, for any $P \unrhd B, B$ Ann $B=0 \subseteq P$,

Received by the editors August 3, 1973.

AMS (MOS) subject classifications (1970). Primary 16A08, 16A12.

Key words and phrases. Essential, injective hull, maximal quotient ring, semiprime, singul ar ideal.

1 This note was written while the author was visiting Leeds University. 
so Ann $B \subseteq P$, implying Ann $B \subseteq B^{\prime}$. Hence $B^{\prime}=$ Ann $B$. Q.E.D.

Lemma 2. Any inessential prime ideal of $R$ is minimal as a prime ideal.

Proof. Let $P, \hat{P}$ be prime ideals of $R$, let $P$ be inessential, and suppose $\hat{P} \subseteq P$. We claim $\hat{P}=P$. Otherwise Ann $P \subseteq \hat{P}$ since $P$ Ann $P=0 \subseteq \hat{P}$. Then $(\text { Ann } P)^{2} \subseteq \hat{P}$ Ann $P \subseteq P$ Ann $P=0$, so Ann $P=0$, contrary to the fact that $P$ is inessential. So we see $P$ is minimal. Q.E.D.

Let $\mathscr{P}_{1}=\{P \in \mathcal{P} \mid P$ is inessential $\}$, let $\mathcal{P}_{2}=\{P \in \mathscr{P} \mid P$ is essential $\}$, and let $J=\bigcap\left\{P \in \mathscr{P}_{1}\right\}$.

Theorem 1. For any $\hat{P}_{\text {in }} \mathscr{P}_{1}, \bigcap\left\{P \mid P \in \mathscr{P}_{1}-\{\hat{P}\}\right\} \neq J$.

Proof. Suppose $\bigcap\left\{P \mid P \in \mathscr{P}_{1}-\{\hat{P}\}\right\}=J$. For any $P \in \mathscr{P}_{1}, \hat{P}$ Ann $\hat{P}=$ $0 \subseteq P$. But $\hat{P} \subseteq P$ by Lemma 2 , so Ann $\hat{P} \subseteq P$. Therefore, Ann $\hat{P} \subseteq \bigcap\{P \mid$ $\left.P \in \mathcal{P}_{1}-\{\hat{P}\}\right\}=J$, so $(\text { Ann } \hat{P})^{2} \subseteq J$ Ann $\hat{P} \subseteq \hat{P}$ Ann $\hat{P}=0$, implying Ann $\hat{P}=0$, contrary to the fact that $\hat{P}$ is inessential. Q.E.D.

Corollary 1. In $R / J, \bigcap\left\{P / J \mid P \in \mathscr{P}_{1}\right\}=0$, but for any $\hat{P}$ in $\mathscr{P}_{1}$, $\bigcap\left\{P / J \mid P \in \mathcal{P}_{1}-\{\hat{P}\}\right\} \neq 0$.

Proof. Immediate.

Call a ring an L-ring if it has a set of primes $\widetilde{\mathscr{P}}$ with intersection 0 , such that for any $\widetilde{P} \in \widetilde{\mathscr{P}}, \bigcap\{P \in \widetilde{\mathscr{P}}-\{\widetilde{P}\}\} \neq 0$. Corollary 1 says any $R / J$ is an $L$-ring. Moreover, if $R$ is an $L$-ring, then for any $\widetilde{P} \in \widetilde{\mathscr{P}}$ it is clear that $0 \neq \bigcap\{P \in \widetilde{\mathscr{P}}-\{\widetilde{P}\}\}=$ Ann $\widetilde{P}$, so each prime in $\widetilde{P}$ is inessential. This yields

Corollary 2. $R$ is an L-ring if and only if $J=0$.

Levy [2] has characterized L-rings as those semiprime rings for which each annihilator (of an ideal) is contained in a maximal annihilator. Moreover, he has shown that the maximal quotient ring of an $L$-ring $R$ is the complete direct product of maximal quotient rings of prime images.

Now let $J^{\prime}=$ Ann $J$. Note that $R / J^{\prime}$ is a semiprime ring. Also $J=$ Ann $J^{\prime}$. (Proof. Clearly $J \subseteq$ Ann $J^{\prime}$. On the other hand, suppose Ann $J^{\prime} \underline{\not} P$ for some $P \in \mathscr{P}_{1} \cdot J^{\prime}$ Ann $J^{\prime}=0 \subseteq P$, so $J^{\prime} \subseteq P$. But $J \subseteq P$, so Ann $P \subseteq$ Ann $J=J^{\prime}$. Hence $(\text { Ann } P)^{2} \subseteq J^{\prime}$ Ann $P \subseteq P$ Ann $P=0$, so Ann $P=0$, implying $P$ is essential. Therefore Ann $J^{\prime} \subseteq \bigcap\left\{P \in \mathscr{P}_{1}\right\}=J$, so $J=$ Ann $J^{\prime}$.)

Call $R$ prime-essential if $\mathcal{P}_{2}=\mathcal{P}$, i.e. all primes are essential. (Since 0 is an inessential ideal, $R$ cannot be prime-essential if $R$ is prime.)

Example. Let $R$ be the ring of all continuous functions from the closed interval $[0,1]$ to the real numbers (with the usual topologies). We claim $R$ is a (semis imple) prime-essential ring. 
Indeed, this ring is quite well known, and it is easy to show, using the compactness of $[0,1]$, that all minimal prime ideals of $R$ have the form $P(t)=\left\{f \in R \mid f^{-1}(0)\right.$ contains an open neighborhood of $t$; i.e. $f$ is "locally" 0 at $t$ \} (which is minimal). To check that $R$ is prime-essential, it suffices to show Ann $P(t)=0$ for all $t \in[0,1]$. Let $f \in$ Ann $P(t)$. We see $f\left(t^{\prime}\right)=0$ for all $t^{\prime} \neq t$ in $[0,1]$, so, by continuity at $t, f=0$. Hence $A n n P(t)=0$, as desired.

As we shall see, being prime-essential is a sort of obstruction to "niceness" of a semiprime ring. Of course, prime-essential rings have essential minimal prime ideals. Also, prime-essential rings have no maximal annihilators (since these are easily seen to be prime and inessential). Hence, any strictly ascending chain of nonzero annihilators can be extended to an infiite chain of annihilators.

Another remark is that each semiprime ring has a prime ideal with no left regular elements. (Proof. The set of left regular elements is a multiplicative set; an ideal maximal with respect to having null intersection with this set is easily seen to be prime.) Hence, every prime-essential ring contains an essential prime ideal with no left regular elements.

Lemma 3. $J^{\prime}=0$ if and only if $R$ is prime-essential.

Proof. $(\Longleftarrow)$ is clear. Conversely, if $J^{\prime}=0$ then $J=A n n J^{\prime}=R$, so $\mathcal{P}_{1}=\varnothing$; therefore $\mathcal{P}_{2}=\mathcal{P}$. Q.E.D.

Theorem 2. $R$ is a subdirect product of the L-ring $R / J$ and the (semiprime) prime-essential ring $R / J^{\prime}$.

Proof. Since $J \cap J^{\prime}=0$, it suffices to prove $R / J^{\prime}$ is prime-essential. Let $\bar{A}=A / J^{\prime}$ be a prime ideal in $\bar{R}=R / J^{\prime}$. For any ideal $\bar{A}^{\prime}=A^{\prime} / J^{\prime}$ in $\bar{R}$ such that $\bar{A}^{\prime} \bar{A}=0$, we have $J A^{\prime} A=0$. It suffices to prove $J A^{\prime}=0$, for then $A^{\prime} \subseteq$ Ann $J=J^{\prime}$, implying $\bar{A}^{\prime}=0$. If $A$ is essential in $R$ then $J A^{\prime}=0$ and we are done. If $A$ is inessential in $R$ then $J \subseteq A$, so $\left(J A^{\prime}\right)^{2} \subseteq J A^{\prime} J \subseteq$ $J A^{\prime} A=0$; hence $J A^{\prime}=0$ (since $R$ is semiprime). Q.E.D.

Incidentally, the above results could be put in a topological framework. Given a subset $\mathscr{P}_{a}$ of $\mathscr{P}$, define $I\left(\mathscr{P}_{a}\right)=\bigcap\left\{P \in \mathscr{P}_{a}\right\}$, a semiprime ideal of $R$, and given an ideal $A$ of $R$, define $V(A)=\{P \in \mathcal{P} \mid A \subseteq P\}$. $\mathcal{P}$ becomes a well-known topological space with closure operation $\overline{\mathcal{P}}_{a}=V\left(I\left(\mathcal{P}_{\alpha}\right)\right)$. There is a 1:1 lattice isomorphism $B \rightarrow V(B)$ between (\{semiprime ideals of $R$ \}, $\cap$ ) and (\{closed subset of $\mathscr{P}\}, \cup$ ), so we identify $B$ with $V(B)$. Lemma 1 says $V(\operatorname{Ann} B)=\overline{\mathcal{P}}-V(B)$; i.e. $B$ is essential if and only if $\mathcal{P}-V(B)$ is 
dense, which means $V(B)$ is nowhere dense. In particular, by Lemma 2, $V(P)$ is nowhere dense if $P$ is a prime ideal which is not minimal prime. $\mathscr{P}_{1}=\{P \in \mathcal{P} \mid V(P)$ has nonempty interior $\} ; R$ is an L-ring if and only if $\mathcal{P}_{1}$ is dense (in $\mathcal{P}$ ).

Application: semiprime rings with left singular ideal 0 . Call a left ideal $B$ of $R$ left essential if $A \cap B \neq 0$ for all nonzero left ideals $A$ of $R$. Note any essential ideal of $R$ is left essential. $Z=\left\{r \in R \mid A n n^{\prime}\{r\}\right.$ is left essential\} is well known to be an ideal of $R$, called the left singular ideal of $R$. In this discussion, we assume $Z=0$. In this case the left injective hull of $R$ can be given a natural multiplication to become a von Neumann regular ring $Q(R)$, called the maximal left quotient ring of $R$ (cf. Faith [1]). If $W$ is an essential ideal of $R$, one can view $W$ as a ring and obtain the following information:

Theorem 3. (i) $W$ is a semiprime ring. If $B$ is a left essential (left) ideal in $R$ then WB is left essential in $W$; if $B^{\prime}$ is a left essential ideal in $W$ then $W B^{\prime}$ is left essential in $R$.

(ii) $W$ bas left singular ideal 0 , and $Q(W)=Q(R)$.

Proof. Straightforward.

Lemma 4. If $A \subseteq B$ are ideals in $R$, and if $B / A$ is left essential in $R / A$, then $B$ is left essential in $R$.

Proof. Clearly $A n^{\prime} B \subseteq A$, so $\left(A n n^{\prime} B\right)^{2} \subseteq\left(A n n^{\prime} B\right) A \subseteq\left(A n n^{\prime} B\right) B=0$, implying $A \mathrm{nn}^{\prime} B=0$. Q.E.D.

Theorem 4. If $A$ and $A^{\prime}$ are ideals of $R$ such that $A=A$ nn $A^{\prime}$ and $A^{\prime}=$ Ann $A$, then the left singular ideals of $R / A$ and $R / A^{\prime}$ are zero, and $Q(R) \approx Q(R / A) \oplus Q\left(R / A^{\prime}\right)$.

Proof. Choose $\bar{r}=r+A \in R / A$ such that $A_{n n}^{\prime}(\bar{r})$ (in $R / A$ ) is left essential, and let $W / A=A n n^{\prime}(\bar{r})$. By Lemma $4, W$ is left essential in $R$. But $W r A^{\prime} \subseteq A A^{\prime}=0$, so $r A^{\prime} \subseteq$ Ann $W=0$. Therefore $r \in$ Ann $A^{\prime}=A$, so $\bar{r}=0$, proving $R / A$ has left singular ideal 0 . Analogously, $R / A^{\prime}$ has left singular ideal 0 .

Next note that $A+A^{\prime}$ is essential because $\operatorname{Ann}\left(A+A^{\prime}\right) \subseteq($ Ann $A) \cap\left(\right.$ Ann $\left.A^{\prime}\right)$ $=A^{\prime} \cap A=0$. Since $A+A^{\prime}=A \oplus A^{\prime}$, we have $Q(R) \approx Q\left(A \oplus A^{\prime}\right)$. Now we claim $A^{\prime} \approx\left(A+A^{\prime}\right) / A$ is essential in $R / A$. Indeed, for any ideal $B / A$ of $R / A$ such that $\left(A+A^{\prime}\right) B \subseteq A$, we have $\left(A+A^{\prime}\right) B A^{\prime}=0$, so $B A^{\prime}=0$ (since $A+A^{\prime}$ is essential). Hence $B \subseteq A n n A^{\prime}=A$, so $B / A=0$, yielding 
the claim. Analogously, $A$ is essential in $R / A^{\prime}$. Applying Theorem 3 again, we conclude

$$
Q(R / A) \oplus Q\left(R / A^{\prime}\right) \approx Q\left(A^{\prime}\right) \oplus Q(A) \approx Q\left(A+A^{\prime}\right) \approx Q(R) \text {. Q.E.D. }
$$

Now Theorem 2 lets us write $R$ as a subdirect product of the $L$-ring $R / J$ and the (semiprime) prime-essential ring $R / J^{\prime}$. By Theorem 4 (since $J=$ Ann $J^{\prime}$ and $J^{\prime}=$ Ann $J$,

$$
Q(R)=Q(R / J) \oplus Q\left(R / J^{\prime}\right) .
$$

Recalling that Levy [2] has shown that the maximal left quotient ring of an $L$-ring is a complete direct product of maximal left quotients of prime images, we have

Corollary. $Q(R)$ is the complete direct product of maximal left quotients of inessential prime images and the maximal left quotient of a prime-essential ring.

The consequences of this corollary for semiprime PI-rings are given in [3]. Briefly speaking, the maximal left quotient of a semiprime primeessential PI-ring has no simple direct summand, whereas the maximal left quotient of a prime PI-ring is simple; hence $J^{\prime}$ is the obstruction to $Q(R)$ being the direct sum of simple rings.

\section{REFERENCES}

1. C. Faith, Lectures on injective modules and quotient rings, Lecture Notes in Math., vol. 49, Springer-Verlag, Berlin and New York, 1967. MR 37 \#2791.

2. L. Levy, Unique subdirect sums of prime rings, Trans. Amer. Math. Soc. 106 (1963), 64-76. MR 26 \#136.

3. L. H. Rowen, Maximal quotients of semiprime PI-algebras, Trans. Amer. Math. Soc. 196 (1974), $127-135$.

DEPARTMENT OF MATHEMATICS, UNIVERSITY OF CHICAGO, CHICAGO, ILLINOIS 60637 